\title{
La Disección In vivo (Vivisección): Una Visión Histórica
}

\author{
In vivo Dissection (Vivisection): A Historical Viewpoint
}

Jorge Eduardo Duque Parra*,**; John Barco Ríos* \& Genaro Morales Parra***

DUQUE, P. J. E.; BARCO R. J. \& MORALES, P. G. La disección in vivo (vivisección): una visión histórica. Int. J. Morphol., 32(1):101-105, 2014.

RESUMEN: La vivisección es un procedimiento exploratorio del ser vivo, que a lo largo de la historia de la humanidad se ha practicado de manera invasiva, tanto en seres humanos como en animales, con el fin de visualizar la morfología y su correlación con la función orgánica. Este procedimiento evolucionó con el descubrimiento y utilización de agentes anestésicos y, posteriormente, con la tecnología de imágenes funcionales no invasivas para el ser vivo, con el propósito de comprender más los aspectos funcionales que los estructurales.

PALABRAS CLAVE: Disección in vivo; Vivisección; Anatomía; Historia.

\section{INTRODUCCIÓN}

En los inicios de la historia humana se consideraba que las enfermedades tenían una causa sobrenatural, y que para su tratamiento sólo se podía recurrir a fuerzas sobrenaturales y confiar en los miembros de la casta sacerdotal. Con el tiempo, los conocimientos sobre la estructura del cuerpo humano se fueron imponiendo poco a poco, despertando la curiosidad de los primeros médicos para guiar la conducta de los más hábiles (Delmas, 1986) en lo quirúrgico. Estos proto-anatomistas constataron la existencia de órganos, cuya forma, ubicación y relaciones anatómicas eran análogas a los observados en los demás seres vivos -especialmente en los vertebrados-. Tales comparaciones fueron, para algunos, motivo de reflexiones e interpretaciones, mientras que otros orientaron este conocimiento a la cura de las enfermedades (Delmas).

Con el paso del tiempo se fue incrementando el estudio meticuloso de la morfología humana, gracias a los trabajos de disección que se hacían en cadáveres y en organismos vivos (vivisección), incluidos los seres humanos. El término vivisección proviene del latín "vivus" (cortar un cuerpo vivo) (Carvalho \& Waizbort, 2010). Probablemente los hombres primitivos se interesaron en la vivisección debido al descuartizamiento que hacían de sus piezas de caza, llegando a conocer mejor la anatomía del animal que la de su misma especie (Delmas).

Nuestros antepasados obtuvieron conocimiento de la anatomía a partir del sacrificio de animales y también de las heridas padecidas por otros seres humanos. Aunque este conocimiento es muy antiguo, sólo se tiene referencia escrita de este saber por las civilizaciones egipcia, alejandrina, griega y romana (O`Dowd \& Philipp, 1994). Para ello, tuvieron que utilizar herramientas variadas para poder disecar los diversos componentes de la morfología animal. Se sabe, por ejemplo, que durante el período alejandrino hubo un progreso en las técnicas de disección que se efectuaban a los criminales condenados (Acierno, 1994).

La palabra disección hace referencia a la voz latina "dissectio onis", que significa división y separación metódica de las partes y órganos del cuerpo para el estudio de su composición (Mascaró y Porcar, 1983; Cadavid Restrepo, 1942). Se asimila disección por anatomía, palabra utilizada desde el tiempo de Hipócrates (460-370 a.C), que proviene de $A v \alpha \tau o \mu$, constituida por el sufijo $\tau$ o $\mu n$ que significa cor-

\footnotetext{
* Departamento de Ciencias Básicas, Programa de Medicina, Facultad de Ciencias para la Salud, Universidad de Caldas, Caldas, Colombia.

** Departamento de Ciencias Básicas Biológicas, Facultad de Salud, Universidad Autónoma de Manizales, Manizales, Colombia.

${ }^{* * * *}$ Facultad de Medicina, Universidad de Manizales, Departamento de Ciencias Básicas Biológicas, Facultad de Salud, Universidad Autónoma de Manizales, Manizales, Colombia.
} 
tar y el prefijo $\alpha v \alpha$ asimilada contemporáneamente por disección de cadáveres, pero el término se ha extendido para incluir el estudio completo de los seres vivos (Clemente, 1985).

También es muy probable que la disección haya tenido sus inicios con las guerras primitivas o en las labores de caza, puesto que durante éstas se afectaban distintas regiones anatómicas de los guerreros y cazadores. Así, muchos de nuestros antecesores practicaron la vivisección o disección in vivo, pero no con un sentido exploratorio para la comprensión morfológica, sino con la intención de destruir a su oponente o a la presa cazada para su alimentación. La vivisección, como método científico, probablemente se inició practicándose con animales, como se infiere por las citas encontradas sobre diversos procedimientos anatómicos; pero también pudo ser motivada por la curiosidad y la especulación fisiológica (García-Albea \& García-Albea, 2006).

Una de estas citas fue hecha por Claudio Galeno (130200) en la parte inicial del libro IX de los procedimientos anatómicos, donde indica que: "La disección que se realiza sobre el animal muerto enseña la posición de cada una de las partes, su número, la peculiaridad de su sustancia, así como su tamaño, forma y composición. La que se realiza sobre los animales vivos enseña a veces directamente su acción y otras veces los supuestos para el descubrimiento de su acción. Es evidente que la disección realizada sobre el animal muerto debe preceder a la que se hace sobre el animal vivo" (Peña Quiñones, 2007). Galeno contribuyó enormemente al conocimiento morfológico, pero sin llegar a abrir un sólo cadáver humano (Sánchez Guisande, 1938); por lo tanto, su conocimiento lo obtuvo de practicar vivisecciones en monos y cerdos (Peña Quiñones). Sus aportes se reconocen en Omnia opera, publicada en Venecia en 1541, en la que se muestra a éste médico Griego, que ejerció en Roma, haciendo una brillante demostración en un cerdo, al que le cortaba el nervio laríngeo recurrente (Gross, 1999), eliminando así la emisión de sonidos, de donde infirió que era el órgano productor de la voz (Gross).

Galeno pensaba, además, que los ventrículos cerebrales contenían algo cuya naturaleza semejaba al aire, pues cuando oprimía la parte posterior del ventrículo, puesto al descubierto en un animal vivo, éste entraba en un estado de rigidez y profundo aletargamiento. De estos experimentos infirió que las lesiones cerebrales sólo afectaban la capacidad de percepción y de movimiento cuando concernían a los ventrículos (Illing, 2002) encefálicos, conceptos que se siguieron aceptando como tales durante muchos siglos, con ligeras modificaciones. Los procedimientos de vivisección continuaron por mucho tiempo. En algunas culturas, como la musulmana, era permitida su realización en cerdos (Garcia albea \& García-Albea), pero no permitían la disección de cadáveres humanos; incluso en Salerno sólo se realizaba una disección al año de un cerdo (Acierno).

Antes de Galeno se hacía vivisección, pues en Omnia opera se menciona que Herófilo de Calcedonia (335-280 a.C) contribuyó a la anatomía haciendo descripción de varias estructuras, entre ellas el calamus scriptorius en el tronco encefálico y los ovarios a los que denominó testis muliebris. Estas observaciones le fueron facilitadas gracias a la liberalidad dada por el rey Ptolomeo, el cual le entregaba condenados a muerte para sus trabajos de vivisección (Falconí Villagómez, 1961). Igualmente, Erasístrato (304-250 a.C), quien disecó unos 600 sujetos, incluyó en sus disecciones algunos individuos vivos antes de ser ajusticiados, lo que le permitió hacer grandes descubrimientos (Delmas), este aspecto de la vivisección de Erasístrato ha pasado a las obras de novela histórica indicándose que fue a ver al rey Ptolomeo y le exigió “...dame personas a las que pueda abrir", aprobando su solicitud con el envío de pastores, los cuales eran llevados por los torturadores quienes veían con horror cómo sus vísceras brotaban por el agujero abierto en sus vientres mientras abrían sus bocas para lanzar gritos desgarradores, pero les embutían algo en ellas a fin de que el médico obtuviera el silencio necesario para trabajar y extirparles las entrañas a sus cuerpos aún vivos. Se cree que Erasítrato anestesiaba a estas personas con meconio (Korber, 2005).

Erasístrato y Herófilo se destacaron entonces como los autores de las principales contribuciones al conocimiento anatómico (Acierno) y, con ellos, los orígenes de la vivisección fueron documentados ampliamente en Alejandría como las primeras disecciones sistemáticas (Moon et al., 2010). Sin embargo, para otros autores, la vivisección no fue adoptada sistemáticamente en términos metodológicos sino hasta el siglo XIX (Carvalho \& Waizbort).

Los conocimientos anatómicos sufrieron un largo estancamiento durante la época del oscurantismo, pues los líderes religiosos de entonces no permitieron las disecciones y mucho menos las vivisecciones. Luego, con la aparición de Andreas Vesalio (1514-1564), figura descollante de la anatomía durante el renacimiento, se encauso de nuevo el curso de la anatomía. Vesalio también practicó la vivisección (Rosetti, 2007), pues además de disecar cadáveres humanos, utilizó animales vivos con el fin de correlacionar la estructura con la función; así lo atestigua el siguiente extracto del capítulo XIX de su libro De fabrica: "Lo que puede aprenderse con la disección del cadáver y con la del ser viviente... La disección de un ser vivo nos muestra claramente a veces la función, y otras nos indica con gran claridad el motivo de la existencia de las diversas parte. Así pues, 
a pesar de que los estudiantes deban adquirir habilidad primero en el estudio de los animales muertos, después, cuando vayan a investigar la acción y el uso de las partes del cuerpo, deberán acostumbrarse al animal vivo. Por otro lado, puesto que muchas partes pequeñas del cuerpo están destinadas a distintos usos y funciones, conviene que nadie dude que las disecciones de animales vivos presenta también muchas contradicciones" (Acierno).

Vesalio fue acusado de impiedad y de asesino ante la inquisición (Patiño, 1989), pues al iniciar la disección del cuerpo de una noble joven que equivocadamente se había dado por muerta, observó con horror que el corazón de dicha mujer aún latía. Fue condenado a muerte por haber practicado dicha "necropsia" in vivo, pero no fue ejecutado, pues Felipe II le salvó la vida a condición de que hiciera un viaje a tierra santa para "purgar sus penas", el cual indudablemente realizó (Sánchez Guisande) para purgar sus penas.

Matteo Realdo Colombo (1516-1559), alumno y sucesor de Vesalio, también practicó la vivisección de animales (Silva, 2009), demostrando que las venas pulmonares contenían sangre en vez de aire y vapores, como se creía anteriormente (Acierno). Seguidamente, William Harvey (1578-1657), con base en vivisecciones de distintos tipos de animales, logró definir un modelo general y lógico sobre la circulación sanguínea sistémica, el cual contradecía las conceptualizaciones anteriores definidas por Claudio Galeno (Silva). Por este mismo período, Marcelo Malpighi (16281694) también ejerció la vivisección, y señaló que el ojo humano no puede ir más allá al observar el animal abierto al relatar que "...ello podía haberme hecho pensar que la sangre escapaba hacia un espacio vacío y era reunida de nuevo en un vaso de recepción por la estructura de las paredes" (Acierno). En un artículo de su época, describió los pulmones y el mesenterio abdominal de los animales viviseccionados (Sin autor, 1981).

René Descartes (1596-1650), sin tener como base las disecciones in vivo, ofreció una imagen mecanicista del flujo de spiritus animalis a través de los ventrículos, tan firme y precisa que se mantuvo por años. En este sentido, Giovanni Borelli (1608-1679) se propuso determinar si la materia que transitaba por el interior de los nervios hacia los músculos era volátil o líquida, y para ello introdujo un animal vivo bajo el agua, el cual lógicamente luchó para no ahogarse. Conforme a la teoría de los spiritus animalis, estos debían haber afluido copiosamente en los músculos estimulados. Después de algunos segundos, Borelli realizó una incisión en un músculo del animal muerto y aún sumergido, pero dado que no ascendió ninguna burbuja a la superficie del agua concluyó que la naturaleza del espíritu vital era acuosa, no gaseosa: un succus nerveus (Illing).
Posteriormente, el anatomista escocés Alexander Monro (1697-1767) realizó cortes transversales de los nervios, pero no encontró en ellos ninguna cavidad; también seccionó los nervios de animales vivos sin ver salir ningún succus nerveus del lugar de la incisión. Luego, ligó los nervios a fin de provocar que el spiritus animalis que fluía por ellos se acumulara en el lado de la barrera más próximo al cerebro, ocasionando de paso una inflamación local de los nervios, pero tampoco esto se verificó (Illing).

A principios del siglo XIX, los fisiólogos Charles Bell and François Magendie reclamaron haber sido los primeros en identificar, de manera separada, las funciones motora y sensitiva de las raíces espinales; esta disputa llevó a dar cuerpo a distintas visiones fisiológicas y al papel de la experimentación, y en ella la vivisección (Berkowitz, 2006). François Magendie (1783-1855) hizo significativas contribuciones en el campo de la neuroanatomía, la fisiología y la farmacología; además, describió una técnica para extraer fluido cerebroespinal para cuantificarlo y describir sus características en pacientes con condición normal y patológica; dilucidó también las funciones de los nervios craneales y precisó la función de las raíces ventrales y dorsales, todo ello haciendo vivisección, así como Bell (Tubbs et al., 2008). El fisiólogo Jean Pierre Marie Fluorens (1794-1867), por ese mismo tiempo, utilizó diferentes animales de laboratorio para separar, pedazo a pedazo, partes del encéfalo y observar cómo se alteraba su comportamiento (Ganten et al., 2005; Duque, 2005). Por ejemplo, tras lesionar el oído interno de palomas en busca de la relación funcional del órgano vestibular (Duque), Flourens esperaba conseguir un trastorno auditivo, pero encontró una afectación del balance postural de tales aves (Berthoz, 2000).

A finales del siglo XIX, el eminente fisiólogo francés Claude Bernard (1813-1878), mediante investigación directa por vivisección en animales (Martínez Briceño et al., 1966; Rodríguez de Romo, 2001), especialmente en perros (Carvalho \& Waizbort; Rodríguez de Romo), y utilizando métodos comparativos, renovó las adquisiciones científicas e impulsó la investigación (Martínez Briceño et al.) en el campo de la Medicina. También en este siglo Rudolf Virchow, quien sentó las bases de la patología celular, reclamaba que la Medicina se debía basar en tres pilares fundamentales: la observación clínica, el estudio anatómico microscópico y la experimentación con animales (Ganten et al.). El zoólogo alemán Karl von Frisch, laureado con el premio Nobel, también realizó vivisección en 1911. Frisch observó que las carpas europeas adoptaban un color oscuro cuando se exponían a la luz, pero la respuesta desaparecía al lesionarles la base del cerebro, lo que le llevó a proponer la existencia de foto-receptores no visuales en la parte profunda del cerebro (Provencio, 2011). 
La vivisección en humanos no sólo fue cosa del pasado remoto, pues en el siglo XX, en Auschwitz, se realizaron experimentos con inyecciones y rayos $\mathrm{X}$ en mujeres judías enfermas de cáncer con el fin de esterilizarlas. En Buchenwald experimentaron con quemaduras de fósforo, hormonas sexuales y edemas provocados por el hambre. En otros campos de concentración nazis, se disparaba balas envenenadas en los muslos a los presos y las víctimas morían a las 2 horas. A otros les inyectaban aire en las venas, petróleo debajo de la piel de ambas piernas y a algunas mujeres se les extirpó los huesos de las piernas. En Buchenwald, el médico del campamento utilizaba en su oficina, como pisapapeles, dos cráneos que procedían de dos judíos transportados de Holanda y que habían sido elegidos por dicho médico por su buena dentadura. El médico les indicó que los requería para unos experimentos y en caso de negarse serían muertos con los demás. A uno de ellos le extrajeron un riñón y al otro el estómago, finalmente les inyectaron bencina en el corazón y después los decapitaron para extraer sus cráneos. Obviamente los nazis negaron haber hecho esto. Durante el juicio de Núremberg contra Heydecker Hermann Goering, comandante de la fuerza aérea alemana, antes de ser sentenciado a la horca dijo: "En 1934 promulgué la ley contra la vivisección. ¿Cómo es posible que yo estuviera en contra de la experimentación con animales y apoyase las torturas de seres humanos? La acusación afirma que yo tenía algo que ver con los escalofriantes experimentos llevados a cabo en algunos campos de concentración bajo el auspicio de la Luftwaffe. Debió de ser Himmler quién ideo esos experimentos estúpidos" (Posada Tamayo, 2006). También se sabe que Goldenshon Mengele seleccionaba prisioneros judíos de los campos de concentración de Auschwitz, en Alemania, para ser utilizados como conejillos de indias en innumerables experimentos, haciendo vivisecciones a sesenta prisioneros diariamente (Posada Tamayo, 2006). El capítulo más oscuro de las investigaciones sobre el cerebro se desarrolló durante esta época del III Reich, en la que algunos investigadores tuvieron participación directa al experimentar con cerebros de enfermos mentales y de impedidos físicos asesinados, siguiendo programas de eutanasia, como fue el caso de Julius Hallervorden del instituto Kaiser Wilhelm de investigaciones del cerebro de Berlín, quien estuvo presente en el asesinato de niños cuyos cerebros terminó diseccionando.

De los indígenas, se sabe que los chibchas hacían el sacrificio de las mojas o guesas, que eran niños de los llanos del Casanare -Colombia-. Cuando entraban en la pubertad los sacrificaban en los templos: "abrían vivo al moja, le sacaban el corazón y las entrañas y le cortaban la cabeza, mientras los músicos cantaban himnos" -esto era entonces una vivisección-. Es probable que antes del sacrificio le administraran al moja una bebida embriagante, como el borrachero (De Zubiría, 1986).

En la actualidad se sigue practicando la vivisección, pero mediante procedimientos quirúrgicos que involucran, muchas veces, el uso de anestesia. Los neuropsiquiatras aprovechan los avances de las modernas técnicas de imagen-RM, SPECT (tomografía computarizada por emisión de fotón único) y PET (tomografía por emisión de positrones)- o funcionales como el electroencefalograma (EEG), video telemetría, etc., para proseguir con el método anatomo-clínico y dar respuesta a enfermedades como la esquizofrenia o la histeria que se resistían a una explicación anatómica o funcional. Así, lo que no logró la simple vista griega al inspeccionar el cerebro, lo que no logró Galeno al ver el encéfalo vivo de los gladiadores cuando eran heridos en el circo romano, lo que no se logró ver con la microscopía, lo han logrado las nuevas técnicas de imagen. Estas técnicas complementan la anatomía de vivisección con la fisiología y nos permiten observar el cerebro en vivo, mientras éste actúa (García Albea \& García Albea).

Actualmente existen controles para la investigación en animales, ya que suponen sufrimiento y muerte para casi un millón de ellos, contando solamente los que se sacrifican en Estados Unidos de Norte América, de ahí que se están empleando estrategias que eliminen el dolor o, más aun, tratar de no recurrir a animales vivos. En el caso de aquellas investigaciones con aplicación en seres humanos, se usarían individuos virtuales que podrían simular la reacción a estímulos biológicos, físicos y químicos, de tal manera que ya no serían necesarios los ensayos con animales (Goldberg \& Hartung, 2006). Sin embargo, el interés en la disección y la vivisección se ha mantenido a lo largo de la historia de la humanidad, y ninguna otra práctica médica ha contribuido más al progreso de la neuroanatomía y de la neurociencia (Moon et al.).

\section{CONCLUSIÓN}

A lo largo de la historia, la vivisección ha sido utilizada como un método científico para la exploración de la estructura anatómica y su función, acabando en muchos casos con la vida de seres humanos y de animales de experimentación. Actualmente se continúa realizando esta práctica de estudio, pero de manera menos invasiva, todo ello con el propósito de lograr una mejor comprensión de la estructura y la función corporal. 
DUQUE, P. J. E.; BARCO R. J. \& MORALES, P. G. In vivo dissection (vivisection): a historical viewpoint. Int. J. Morphol., 32(1):101-105, 2014.

SUMMARY: Vivisection is an exploratory process of being alive, that throughout the history of mankind has been performed invasively, in both humans and animals, in order to visualize the morphology and its correlation with organ function. This procedure has evolved with discovery and use of anesthetic agents and, functional subsequently functional imaging technology for noninvasive living being, with the aim of understanding more functional than structural aspects.

KEY WORDS: Vivisection; Anatomy; History.

\section{REFERENCIAS BIBLIOGRÁFICAS}

Acierno, L. J. The history of cardiology. Basel, Editions Roche, 1994.

Berkowitz, C. Disputed discovery: vivisection and experiment in the $19^{\text {th }}$ century. Endeavour, 30(3):98-102, 2006.

Berthoz, A. The Brain's sense of movement. Cambridge, Harvard University Press, 2000.

Cadavid Restrepo, T. Raíces griegas y Latinas. Etimologías médicas y biológicas. Bogotá, Academia Colombiana de la Lengua, 1942.

Carvalho, A. L. \& Waizbort, R. Pain beyond the confines of man: a preliminary introduction to the debate between Frances Power Cobbe and the Darwinists with respect to vivisection in Victorian England (1863-1904). Hist. Cienc. Saude Manguinhos, 17(3):577605, 2010.

Clemente, C. D. Anatomy of the human body. Baltimore, Williams \& Wilkins, 1985.

De Zubiría, R. La medicina en la cultura Muisca. Bogotá, Empresa editorial, 1986.

Delmas, A. La anatomía Humana. Barcelona, Paidotribo, 1986.

Duque, P. J. E. Historia de los conceptos sobre la estructura y función del sistema vestibular y su evolución en vertebrados e invertebrados. Sevilla, Universidad Pablo de Olavide, 2005.

Falconí Villagómez, J. A. Asclepio y Cronos. Páginas de la historia médica y paramédica. Guayaquil, Casa de la Cultura Ecuatoriana, 1961.

Ganten, D.; Deichmann, T. \& Spahl, T. Vida, Naturaleza y ciencia. Todo lo que hay que saber. México, Taurus, 2005.

García-Albea, R. E. \& García-Albea, M. J. Cerebro, mente y síntoma. Rev. Neurol., 42(7):439-43, 2006.

Goldberg, A. M. \& Hartung, T. Pruebas toxicológicas con animales. Investigación y Ciencia, 354:68-75, 2006.

Gross, C. G. Brain, Vision, memory. Tales in the history of Neuroscience. London, The MIT Press, 1999.

Illing, R. B. De la trepanación a la teoría de la neurona. Mente y Cerebro, 1:82-9, 2002.

Korber, T. El médico del emperador. Barcelona, Ediciones B, 2005.
Martínez Briceño, R. \& Hernández de Alba, G. De Hipócrates a Pasteur. Historia de la medicina colombiana. Bogotá, Ediciones Sol y Luna, 1966.

Mascaró y Porcar, J. M. Diccionario terminológico de ciencias médicas. Barcelona, Salvat editores, 1983.

Moon, K.; Filis, A. K. \& Cohen, A. R. The birth and evolution of neuroscience through cadaveric dissection. Neurosurgery, 67(3):799809,2010

O'Dowd, M. J. \& Philipp, E. E. Historia de la ginecología y obstetricia. Barcelona, Edikamed, 1994.

Patiño, J. F. Andreas Vesalio. Tribuna Médica, 80:255-65, 1989.

Peña Quiñones, G. Galeno de Pérgamo y las Ciencias Neurológicas. Medicina, 29(1):34-9, 2007.

Posada Tamayo, S. Médicos siniestros. Sinbata, 4:10-4, 2006.

Provencio, I. Un órgano oculto en los ojos. Investigación y Ciencia, 418:33-7, 2011.

Rodríguez de Romo, A. C. El juego experimental de Claude Bernard. Elementos, 42:29-32, 2001.

Rosetti, S. El cerebro de Leonardo. Buenos Aires, Grupo Editorial Lumen, 2007.

Sánchez Guisande, G. Datos para la historia de la anatomía en España. Buenos Aires, Publicaciones de la cátedra de la historia de la medicina, Universidad de Buenos Aires, 1938. pp.77-128.

Silva, J. M. Da descoberta da circulação sanguínea aos primeiros factos hemorreológicos. Rev. Port. Cardiol., 28(11):1245-68, 2009.

Sin autor. Marcelo Malpighi: Una vida de exploración apasionada. Consulta, 8:19-22, 1981.

Tubbs, R. S.; Loukas, M.; Shoja, M. M.; Shokouhi, G. \& Oakes, W. J. François Magendie (1783-1855) and his contributions to the foundations of neuroscience and neurosurgery. J. Neurosurg., 108(5):1038-42, 2008.

Dirección para Correspondencia:

Jorge Eduardo Duque Parra

Programa de Medicina

Universidad de Caldas

Recibido : 31-03-2012

Manizales - COLOMBIA

Aceptado: 19-11-2013

Email: jorge.duque_p@ucaldas.edu.co 